\title{
Electronics for the LAMPF Neutrino Experiment's Veto Counter System
}

\author{
Charlie Dalton
}

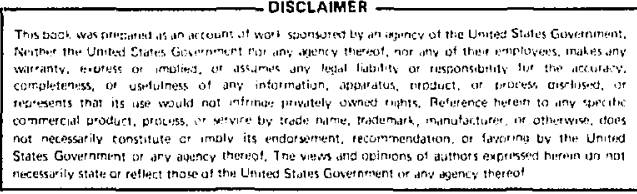




\title{
ELECTRONICS FOR THE LAMPF NEUTRINO EXPERIMENT'S
}

VETO COUNTER SYSTEM

\author{
by
}

Charlie Dalton

\begin{abstract}
A cosmic-ray veto detector has been constructed in the neutrino cave at Los Alamos National Laboratory. This report describes the electronic readout system designed to buffer and compact the data from the detector.
\end{abstract}

\section{INTRODUCTION}

An essential feature of any neutrino experiment is the cosmic-ray veto system. This report describes the electronics for the system being installed for an experiment at LAMPF to measure $v$-e elastic scattering. The very small cross section for $v$ scattering means that the detector must be very large and the surrounding veto system must have a high efficiency. Although the electronics have been designed for a specific experiment, several features may be of general interest.

For the LAMPF experiment, the detector consists of a sandwich of flash chambers and scintillators with a cross section of $3 \mathrm{~m} \times 3 \mathrm{~m}$ and a thickness of $4.5 \mathrm{~m}$. The detector is housed in a cave consisting of iron and heavy concrete. The inside dimensions of the cave are $5.8 \mathrm{~m} \times 5.8 \mathrm{~m} \times 5.8 \mathrm{~m}$ (see Fig. 1).

The veto system consists of 550 drift-tube counters that line the inside of the cave and the associated electronics. There are four layers on the sides and top and a single layer on the floor. An additional $15 \mathrm{~cm}$ of iron shielding are placed between the drift-tube layers and the central detector.

The first defense against cosmic rays is provided by the iron and heavy concrete shielding. Those particles with sufficient energy to penetrate the outer shield are detected by the drift tubes. The electronic system must process signals from the detectors and allow flexibility in the logic during the course of the experiment. For instance, a stronger coincidence requirement between layers reduces the deadtime of the system but adversely affects the efficiency, A rather important feature of the system is the ability to maintain a time history of cosmic-ray triggers. Then when an event occurs in the central detector, the preceding time bins can be examined to see if the event could have come from the decay of a cosmic-ray muon that entered the detector earlier. 
With the complexity of the system, the need for reliability, and the long time period over which the experiment will operatt. adequate testing procedures are important. These testing capabilities have been designed into the system and are computer controlled.

\section{DRIFT TUBES}

The drift tubes have been designed to be rugged, reliable, and inexpensive. Each unit is $20.3 \mathrm{~cm}$ wide, $4.8 \mathrm{~cm}$ high, and is formed from an aluminum extrusion consisting of eight triangular cells (see Fig. 2). The 1ength of the extrusion varies between 2.7 and $5.8 \mathrm{~m}$, depending on its location in the system.

At the end of each extrusion, a $1.27-\mathrm{cm}-\mathrm{radius}$ notch is cut in every other inner web, which allows a gas mixture to flow down one triangle and up the next. With a gas inlet on one side and an outlet on the opposite side, the gas will flow in a serial fashion through the complete counter.

The ends of the extrusions are sealed by $0.63-\mathrm{cm}-\mathrm{thick}$ aluminum plates that include eight feedthroughs with solder lugs centered on the triangles. One end has the gas inlet, outlet, and a box to house the preamplifier, discriminator, and high-voltage filter (see Fig. 3).

A 45- $\mathrm{mm}$ gold-coated tungsten wire is strung down each triangle, the feedthrough aligns the wire, and a tension of $350 \mathrm{~g}$ is applied. Epoxy is used to seal the opening around the wire and hold the wire under tension. To ensure tension and make electrical contact, the wire is wrapped around the solder lugs on each end and soldered. On the preamplifier end, the eight signal wires are connected to form one input to the preamplifier.

A positive high voltage $(\sim 2.5 \mathrm{kV})$ is applied to the signal wires. When a charged particle traverses the active volume of the drift tubes, some of the gas is ionized and the free electrons are attracted toward the sense wire. These electrons avalanche in the vicinity of the sense wire, producing a small signal that can be amplified and detected.

Because of the large size and triangular cross section of the drift tubes, the signals coming from the drift tubes are far from ideal, and the electronics must compensate to avoid problems.

The amplitude of the signals varies widely because particles can traverse the active volume at any angle, and even for normal incidence, the ionized path in the corner of the triangular-shaped cells is much shorter than in the niddle next to the signal wire. The arrival time of signals varies up to $400 \mathrm{~ns}$ because of the drift velocity of the electrons through the gas. Some signals have long tails caused by particles that traverse the drift tube at a shallow angle to the signal wire. Also, as an economy measure, only one amplifier is used per drift tube, which means that the eight triangular cells look like eight improperly terminated signal sources. Large amplitude pulses can reflect down the cells and cause mu1tiple pulses out of the electronics.

\section{FRONT-END ELECTRONICS}

The signal from the drift tube arrives first at the preamplifier (Fig. 4) and discriminator (Fig. 5). These elements convert the raw signal into a standard level that can be sent over long distances and then processed with standard digital logic. The preamplifier and discriminator are made on separate boards, to give the very fast digital signals and large impulse loads on the power supply a different ground return from the discriminator, and to form part of a Faraday cage shield around the sense wires. 
The preamplifier board contains the high-voltage circuit that removes unwanted spikes and 1imits the current to the signal wires. The signal is removed from the high-voltage signal wires by ac-coupling into a wide-band video amplifier with input diode protection in case of high-vo. age transients. The video amplifier is a current-to-voltage converter with a transfer resistance of $\approx 1 \Omega$. The output signal to the discriminator board is carried via a differential pair of lines to a fast emitter-coupled logic (ECL) comparator.

The discriminator is a voltage comparator with a threshold range of 10 to $200 \mathrm{mV}$. Ferrite beads are used to slow down the leading edges of the input pulses to reduce the amount of feedback to the preamplifier. The output is a singleended ECL time-over-threshold pulse. The use of single-ended outputs, rather than balanced, was a compromise between the cost and isolation offered by coaxial cable and twisted pair. These cables are located in an environment with a high level of radio frequency interference (RFI) as operated by the flask chamber system. The discriminator board also contains the low-voltage power circuit that filters the $\pm 7 \mathrm{~V}$ and drops the voltage to \pm 5.2 , which allows multiple drift tubes to be daisychained together without the worry of voltage drops down the power line.

\section{READOUT EIECTRONICS}

The readout collects data from all drift tubes, processes and compacts the data, and makes the information available to both the computer system and the fast logic of the experiment. The electronics are located in a chassis with capacity for 736 input lines and in two auxiliary CAMAC modules. Figure 6 shows a block diagram of the readout system.

The output signal from each of the drift tubes arrives via 32-m-long RG-174 cables. The signals are first converted to transistor-transistor logic (TTL) using an ECL-to-TTL translator. Next comes a gating circuit, used to inhibit the input signal during testing of the readout system and to discriminate against double pulses. Then the signal triggers a one-shot set at $600 \mathrm{~ns}$. The width of 600 ns was chosen to give a minimum overlap of $200 \mathrm{~ns}$ for a particle that hits a wire in one tube and the corner of the next tube.

The output of the 600-ns one-shot goes to four places: 1) a connector on the rear of the chassis to be used by the user in his custom electronics, 2) programmed array logic (PAL), which allows the user to have a fast veto of cosmic rays for his experiment, 3) time-history storage, compaction, and readout, and 4) hold-off circuit.

The hold-off circuit will make sure the readout and programmed array logic do not react to double pulses caused by particles that traverse the signal wire at shallow angles. This is accomplished by triggering a one-shot set at $2 \mu s$, which inhibits the input signal through the gating circuit. With this procedure, the hold-off one-shot can be enabled or disabled with a jumper allowing the different experiments to detect double pulses if desired.

The PAL forms an $O R$ of all tubes in a given layer of drift tubes. Then independent coincidences are formed between all combinations of layers in a wall. The outputs correspond to all levels of coincidence that are triggered. The possibilities are four out of four, three out of four, two out of four, and one out of four signals. A second set of outputs is available that only gives the highest level of coincidence out. The PAL circuit is duplicated for each wall, floor, and roof. This allows the user to decide the level of efficiency versus deadtime for his experiment. The highest efficiency for the veto system occurs when any one out of four layers is accepted as a veto. For each trigger of the veto system, the experiment will be gated off for 10 to $20 \mu s$ to protect against 
decaying muons. If the resulting deadtime is higher than desired, the next level of coincidence is available. Figure 7 shows the truth table for the different possibilities of hits in each layer and what outputs will be valid.

Another destination for the signals from the drift tubes is to an 80-deep static shift register that forms the memory for the time history. The memory is 80-bits deep for each wire and, when clocked every 500 ns by an externaj clock, will give a resolution of $\pm 500 \mathrm{~ns}$ for $-40 \mu \mathrm{s}$. During data collection, the registers will work like a push-down stack memory that retains the most recent 80 bits of information. When an event pulse is issued by the user, the external clock is gated off and the rotate bit is enabled on the shift register, making it work as a circular buffer. The registers now contain up to 80 bits of information for each wire. After the rotate bit is toggled, the bits corresponding to the oldest time slot are read into a parallel-to-serial (P/S) register. When the $\mathrm{P} / \mathrm{S}$ register is clocked, the last input and drift tube (DT) counters are also advanced to keep track of which drift tube is being read. A second counter called the time counter keeps track of what time slot is being read. The $P / S$ register is shifted out to a coincidence gate with an internal clock to detect a high level to indicate a bit in one of the drift tubes. During this time, the internal clock has been advancing the DT counter and when a hit is detected, the clock is disabled and the counter number and time number are loaded into a first-in-first-out (FIFO) memory and the clock is enabled after completing memory load. With this procedure, tubes without data will not be read into memory. When the last bit of the $\mathrm{P} / \mathrm{S}$ register is detected, the time counter is advanced one and the bits of information from the next time slot are loaded into the $\mathrm{P} / \mathrm{S}$ registers and clocking starts over. This is repeated until all bits of the 80 -deep registers have been read. Since most bits are expected to be zero, the compaction factor is large and the essential information is ready for efficient transfer to a computer via CAMAC. Actually, after the first word is stored in the FIFo memory, the computer reads can overlap storage in this memory buffer. To test if the module is ready for readout, a Test $\mathrm{Lam}$ is issued and if $Q=1$, valid data are available. Then a read command is issued and the first word is read out with $Q=1$ if data were present. The read command is issued until all data have been read, indicated by $Q=0$. Table I gives all the CAMAC commands.

\section{TABLE I}

\section{CAMAC COMMANDS}

$\begin{array}{ll}\text { F2A0 } & \text { READ one word from memory, } Q=1 \text { if valid data } \\ \text { F8A0 } & \text { Test Lam, } Q=1 \text { if Lam set } \\ \text { F9A0 } & \text { Clear module for event } \\ \text { F24A0 } & \text { Clear end word counter } \\ \text { F16A0 } & \text { Write end word into module } \\ C \text { or } Z & \text { Clears module } \\ X=1 & \text { Is returned for a11 valid commands }\end{array}$

Since all 80 time slots are read each time, if an event trigger occurs within $40 \mathrm{\mu s}$ of enabling the system after a previous event, the extra time slots must be discarded by the user.

The readout system is designed to accommodate extra inputs in case cracks are found between the walls or ceiling and counters have to be added. The user will tell the system the number of inputs in use. This is stored in the last input register and is loaded from CAMAC. 
The compare register will compare the number in the last input register to the last Input counter to tell the system when it has read the last bit of the 80-deep register for each time slot. When the last time slot has been read, the user will issue a command that will clear and reset the system for the next event.

\section{TESTING FOR THE ELECTRONICS}

A test module has been designed to allow the user to check out the readout system by loading bits of information into the 80-deep registers for readout. The user will have the choice of two different test procedures. The first procedure will let him set a bit pattern to simulate the inputs from the drift tubes. The inputs will be gated off during all testing. This bit pattern will then be clocked into the gating system and one-shots continually until the user decides to stop the test. This test will allow the PAL fast logic to be checked with a scope and also issue pulses to the user port.

The second procedure will set a bit pattern, but the test module will issue only 80 clock pulses. This loads the bit pattern into the 80 -deep registers. Then a fake event pulse is generated and normal readout started. This allows checkout of the 80 -deep registers, P/A registers, ant the FIFo memory.

\section{TESTING OF DRIFT TUBES}

Before the preamp-discriminator is installed, each signal wire is checked for continuity and resistance to be sure a wire is not shorted or broken. Next, the wires are connected to the buss Iine and a capacitance reading is made to ensure all the wires are connected. Then the drift tube is filled with a noble gas and high voltage applied while monitoring the current. If the tube does not respond correctly, the high voltage is reversel with air being the gas filler. This process cleans off the wire. The drift tube is then refilled with a noble gas and high voltage applied. If the tube passes the high voltage holdoff test, the preamp-discriminator is Installed, and the single's rate and efficiency plateau is measured. The drift is now ready for installation in the cave.

\section{ACKNOWLEDGEMENTS}

I would like to thank the experimenters on the Neutrino Electron Elastic Scattering experiment for their discussions and ideas. I would especially like to thank Vernon D. Sandberg (MP-4) for help with the preamplifier-discriminator and Hans-Peter Von Gunten for the PAL system.

\section{APPENDIX}

The power requirement for main readout will be $w 80.0 \mathrm{~A}$ of $+5.0 \mathrm{~V}, 7.4 \mathrm{~A}$ of $-5.2 \mathrm{~V}, 18.0 \mathrm{~A}$ of $-12.0 \mathrm{~V}$, and $3.08 \mathrm{~A}$ of $-12.0 \mathrm{~V}$. The control module demands $\approx 0.9 \mathrm{~A}$ of $+5.0 \mathrm{~V}$. 


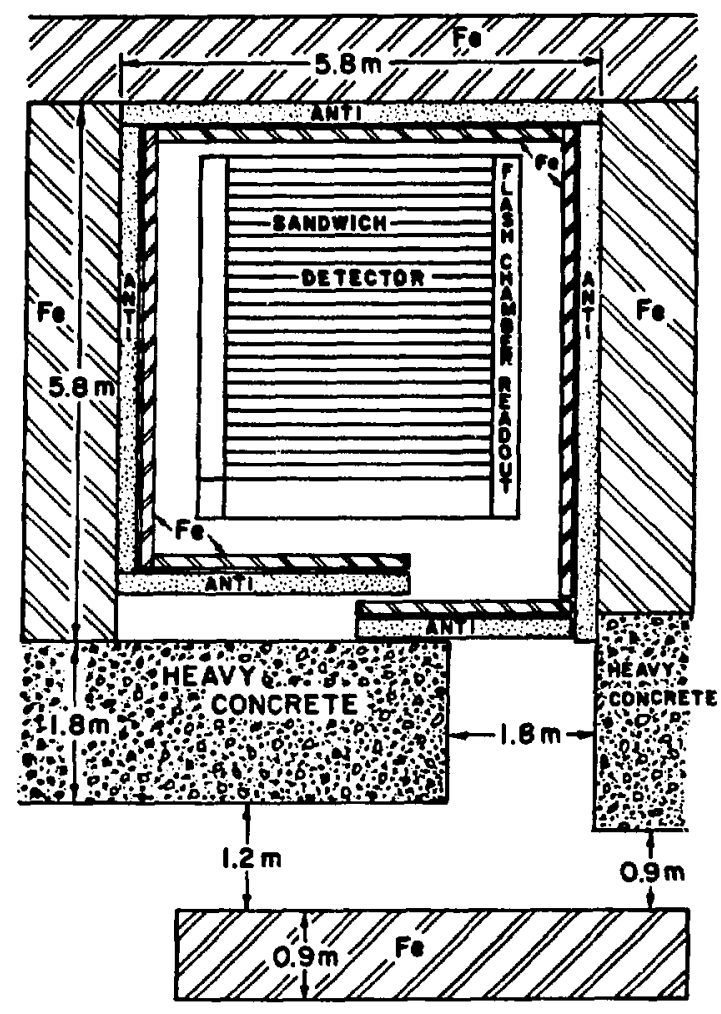

Fig. 1. Top view of LAMPF neutrino cave with detector.

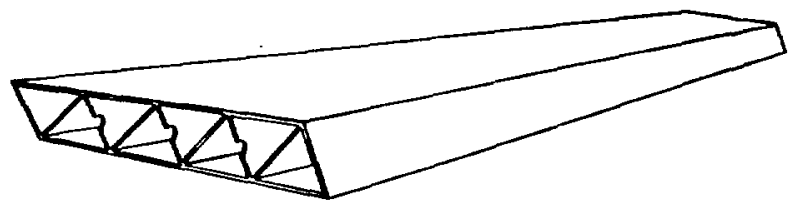

Fig. 2. Aluminum drift tube extrusion.

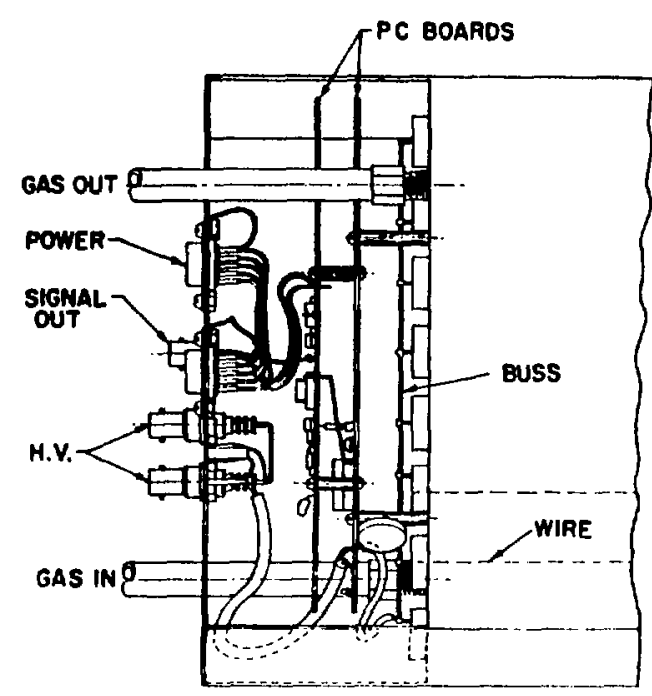

Fig. 3. View of electronic end of drift tube.

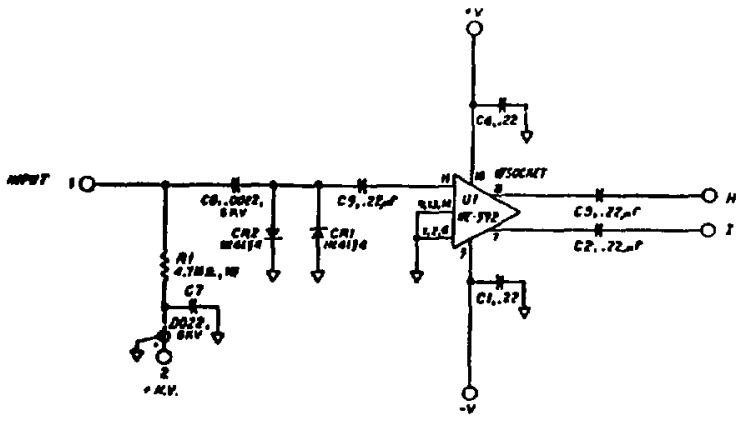

Fig. $\rightarrow$ Schematic of preamplifier. 


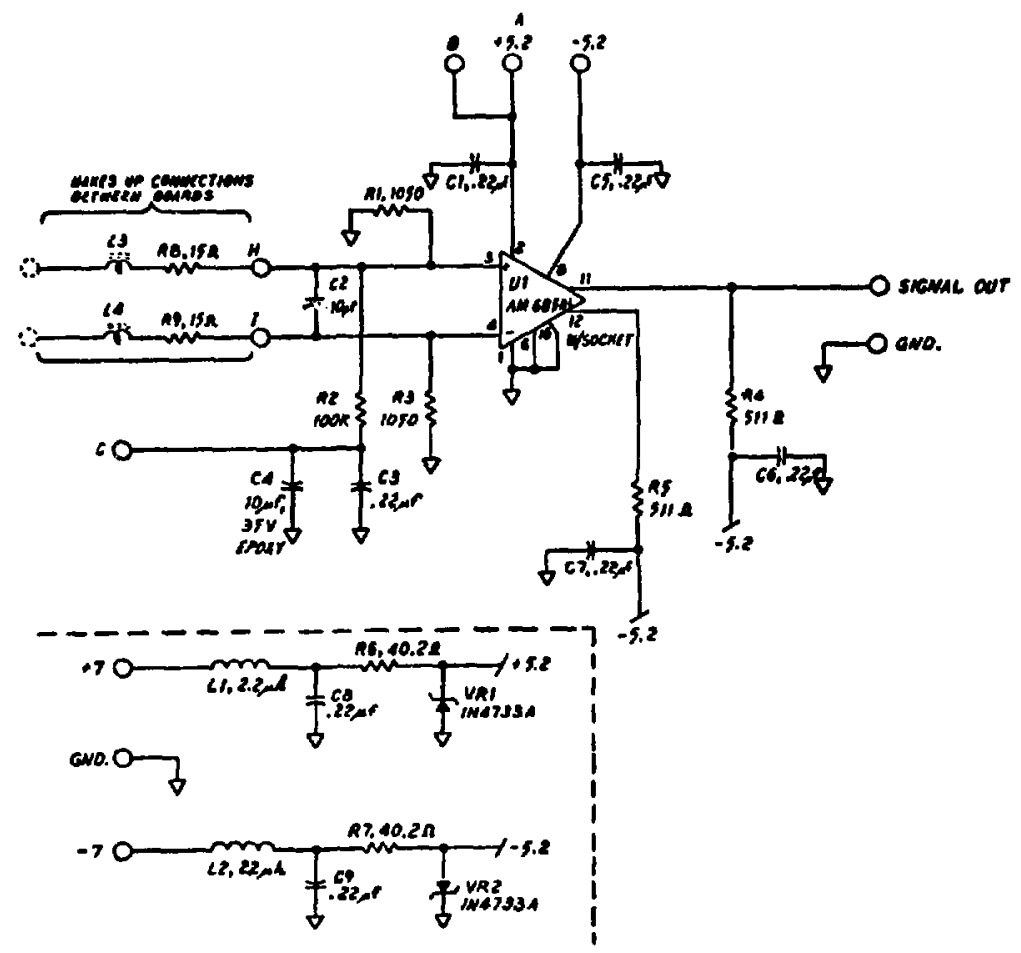

Fig. 5, Schematic of discriminator.

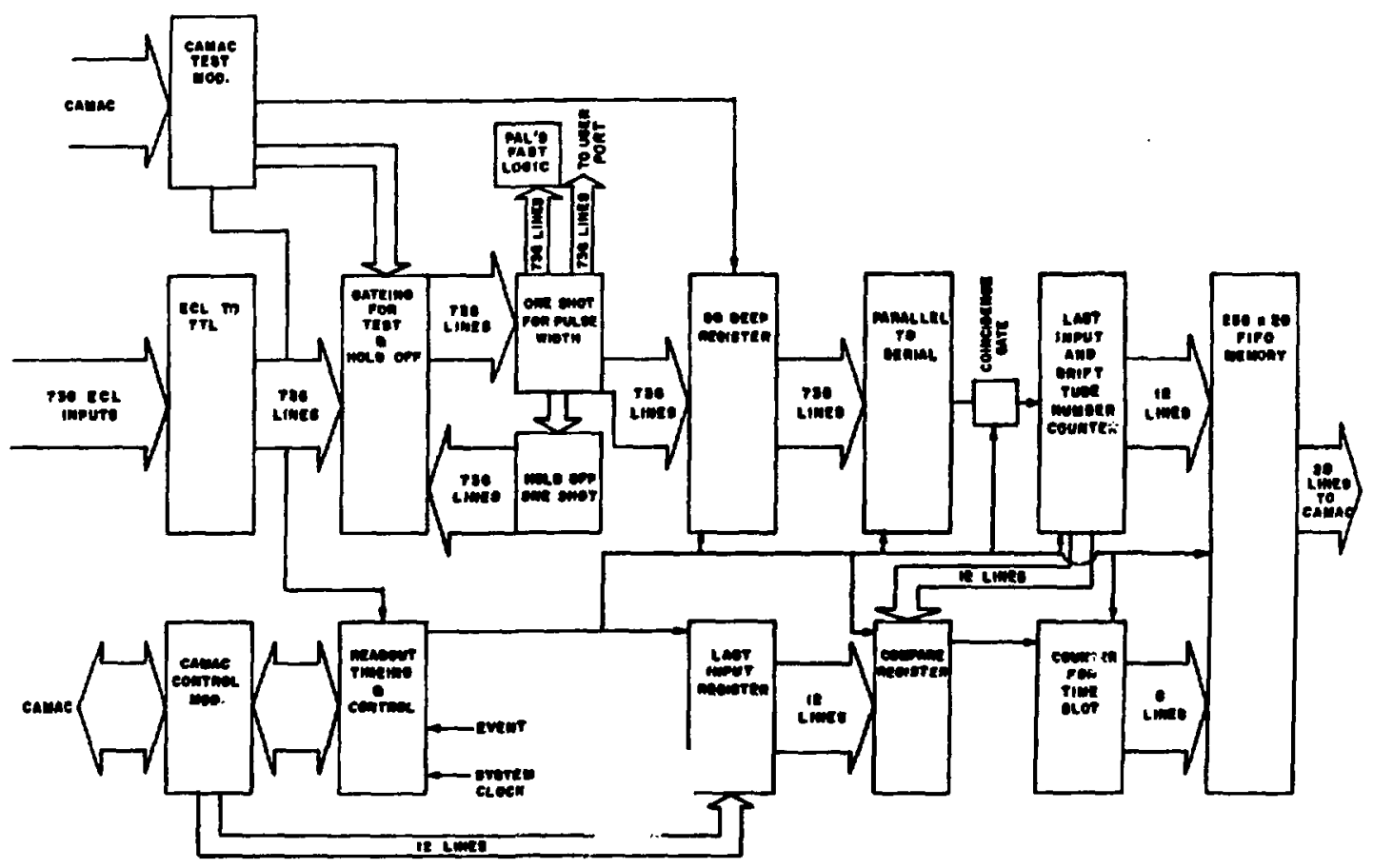

Fig. 6. Block diagram of the readout system. 


\begin{tabular}{|c|c|c|c|c|c|c|c|c|c|c|c|c|}
\hline \multirow[t]{2}{*}{ Possibilities } & \multicolumn{4}{|c|}{$\begin{array}{c}\text { Drift Tube } \\
\text { Layer } \\
\end{array}$} & \multicolumn{4}{|c|}{$\begin{array}{c}\text { Coincidence } \\
\text { Outputs } \\
\end{array}$} & \multicolumn{4}{|c|}{$\begin{array}{c}\text { Highest Level } \\
\text { Coincjdence } \\
\text { Outputs } \\
\end{array}$} \\
\hline & $\underline{1}$ & $\underline{2}$ & $\underline{3}$ & $\underline{4}$ & $1 / 4$ & $2 / 4$ & $3 / 4$ & $4 / 4$ & $1 / 4$ & $2 / 4$ & $3 / 4$ & $4 / 4$ \\
\hline 1 & $x$ & $x$ & $x$ & $x$ & $\mathrm{X}$ & $X$ & $\mathrm{X}$ & $X$ & & & & $x$ \\
\hline 2 & $x$ & $x$ & $\mathrm{X}$ & & $X$ & $x$ & $x$ & & & & $X$ & \\
\hline 3 & $\mathrm{X}$ & $X$ & & $\mathrm{X}$ & $X$ & $X$ & $x$ & & & & $x$ & \\
\hline 4 & $x$ & & $x$ & $x$ & $x$ & $x$ & $\mathrm{X}$ & & & & $X$ & \\
\hline 5 & & $\mathrm{X}$ & $X$ & $x$ & $x$ & $x$ & $x$ & & & & $x^{\prime}$ & \\
\hline 6 & $X$ & $\mathrm{X}$ & & & $x$ & $x$ & & & & $x$ & & \\
\hline 7 & $x$ & & $\mathrm{X}$ & & $x$ & $x$ & & & & $x$ & & \\
\hline 8 & $x$ & & & $x$ & $X$ & $\mathrm{X}$ & & & & $x$ & & \\
\hline 9 & & $x$ & $x$ & & $x$ & $x$ & & & & $\mathrm{X}$ & & \\
\hline 10 & & $\mathrm{x}$ & & $\mathrm{x}$ & $\mathrm{X}$ & $\mathrm{x}$ & & & & $\mathrm{x}$ & & \\
\hline 11 & & & $x$ & $x$ & $x$ & $X$ & & & & $X$ & & \\
\hline 12 & $\mathrm{X}$ & & & & $\mathrm{X}$ & & & & $\mathrm{X}$ & & & \\
\hline 13 & & $\mathrm{X}$ & & & $\mathrm{X}$ & & & & $X$ & & & \\
\hline 14 & & & $X$ & & $x$ & & & & $X$ & & & \\
\hline 15 & & & & $\mathrm{X}$ & $x$ & & & & $x$ & & & \\
\hline
\end{tabular}

Fig. 7. Truth table for the different possibilities of hits. 